\title{
Bone marrow failure syndromes, a practical approach to diagnosis
}

\author{
Carlos Cantu $^{1} \cdot$ Maria A. Proytcheva ${ }^{1}$
}

Received: 29 June 2015 / Accepted: 7 July 2015 / Published online: 6 August 2015

(C) Springer-Verlag Berlin Heidelberg 2015

\begin{abstract}
The inherited bone marrow failure (IBMF) syndromes are a heterogeneous group of disorders with characteristic quantitative or qualitative abnormalities affecting one or more hematopoietic lineages. IBMF syndromes are due to germline mutations affecting structural proteins or key cellular pathways such as DNA repair, telomerase biology, and ribosomal biosynthesis. These mutations lead to single or multiple peripheral blood cytopenias that either result from an absence of one or more lineages of hematopoietic progenitors in the marrow or to an increase cell death of one or more marrow progenitor lineages. Most IBMF syndromes manifest in childhood, but some are recognized later in life depending on the severity of symptoms. This review we will summarize the clinical presentation, diagnostic findings, and genetic findings of the most frequent and best studied IBMF syndromes with a special focus on the diagnostic dilemmas which can occur during the work up of a child with suspected a IBMF syndrome.
\end{abstract}

Keywords Inherited bone marrow failure syndromes .

Anemia $\cdot$ Neutropenia $\cdot$ Pancytopenia $\cdot$ Pediatric $\cdot$ Diagnosis

\section{Introduction}

A common feature of all inherited bone marrow failure (IBMF) syndromes is the presence of quantitative or qualitative abnormalities affecting one or more of the erythroid, my-

Maria A. Proytcheva

mproytcheva@pathology.arizona.edu

1 University of Arizona, Banner University Medical Center, Tucson, AZ, USA eloid, or megakaryocytic lineages leading to anemia, leukopenia, thrombocytopenia, or pancytopenia. This is a heterogeneous group of rare disorders and the diagnosis, almost always challenging, is usually established overtime. There is a significant overlap between the clinical and, to some degree, hematologic manifestation of various IBMF syndromes. In addition, while some solely present with hematologic abnormalities and complications due to the peripheral cytopenias, others have a high incidence of skeletal abnormalities as well as an increased risk of hematologic malignancies and/or solid tumors.

Genetically, the IBMF syndromes are heterogeneous too and result from germline mutations affecting various key cellular pathways such as ribosomal biogenesis, telomerase biology, DNA repair, and the function of structural proteins. There is no consistent genotypephenotype correlation. For example, mutations affecting the same cellular pathway-i.e., ribosome biosynthesis - can result in different phenotypes - either as pure red cell aplasia in Diamond Blackfan anemia, neutropenia Shwachman-Diamond syndrome, or progressive bone marrow failure in $\mathrm{X}$-linked dyskeratosis congenita. Furthermore, the same phenotype-i.e., anemia with reticulocytopenia - can result from mutations affecting proteins involved in ribosomal biosynthesis (Diamond Blackfan anemia) as well as mutations in various structural proteins (Congenital Dyserythropoietic anemias). Since these syndromes have different prognosis and various risk of developing hematologic or solid organ malignancies, knowledge of the clinical and laboratory manifestation(s) is essential to the success of establishing an accurate diagnosis and proper management. To facilitate this process, we will review the most frequent IBMF syndromes and provide a practical 
hematopathology approach to establishing and confirmation of the diagnosis.

\section{Bone marrow failure syndromes associated with pure red cell aplasia}

\section{Diamond Blackfan anemia}

The most frequent cause of inherited isolated red blood cell production failure is Diamond Blackfan anemia (DBA). A majority of patients present with transfusion dependent anemia in the first year of life, although the affected can manifest later in life and such cases are considered non-classical DBA. In addition to anemia, congenital abnormalities can be seen in approximately half of patients with DBA including dysmorphic faces, short stature, eye, kidney, and hand abnormalities [1]. DBA is also associated with an increased risk of malignancies including acute myeloid leukemia (AML), myelodysplastic syndrome (MDS), colon cancer, female genital cancer, and osteosarcoma [2].

The diagnosis of DBA can be challenging. The typical presentation includes macrocytic anemia and reticulocytopenia in the first year of life. The peripheral blood film review is essential in the diagnostic process, and it provides clues to the accurate diagnosis (Fig. 1). It shows macrocytic normochromic red blood cells with no significant anisocytosis and polychromasia. The bone marrow (BM) is normocellular and shows a paucity of erythroid precursors relative to the overall intact granulopoiesis and megakaryopoiesis. Thus, the anemia in DBA is a result absolute erythroid hypoplasia and an overall loss of erythroid mass. Other laboratory abnormalities include an elevated erythrocyte adenosine deaminase (eADA) activity which is considered diagnostic since its specificity and sensitivity when significantly elevated is high ( 84 and $95 \%$, reps.). Mild increase in eADA is non-specific. The DBA laboratory findings are summarized in Table 1 and include increase fetal hemoglobin, strong expression of $\mathrm{i}$ antigen on the red blood cells, and increased folate and vitamin B12 levels. In addition to anemia, rare patients with DBA may present with neutropenia, thrombocytopenia, or thrombocytosis. Since the anemia in DBA is due to decreased production rather than increased destruction, there is no accumulation of hemoglobin degradation products.

The differential diagnosis is somewhat different depending on the age of presentation. In the first year of life, the differential diagnosis includes other bone marrow failure syndromes such as congenital dyserythropoietic anemia (CDA), Pearson syndrome, hemoglobinopathies, hereditary hemolytic anemias, and immune mediated hemolytic anemia. Bone marrow studies are necessary to distinguish between DBA, CDA (described below), or other disorders. Pearson syndrome manifests with vacuolation of the marrow precursors and increased numbers of ring sideroblasts, features not seen in DBA. Characteristic features of the hereditary hemolytic anemias such as increased peripheral red cell destruction leading to reticulocytosis and increased hemoglobin degradation resulting in splenomegaly are not seen in DBA. In older children, the differential diagnosis includes various acquired causes of anemia such as transient erythroblastopenia of childhood as well as parvovirus B19 infection, human immunodeficiency virus (HIV), or other infection(s), drug-induced, and/ or immune mediated red cell aplasia (Table 2). In those acquired causes, a review of the patient's history will show no significant anemia prior to the onset of disease.

While most cases are sporadic, DBA is considered a genetic disease with a mixed pattern of inheritance including autosomal dominant, autosomal recessive, and possibly X-link form [3]. DBA is associated with distinct mutations of the genes participating in the ribosome biosynthesis pathway and is the prototype of a group of disorders called "ribosomopathies" such as Shwachman-Diamond syndrome and X-linked dyskeratosis congenita (described below). Ribosomes are essential for the function of the cells, and their biogenesis is a complex and highly regulated process requiring structural ribosomal RNAs, approximately 79 ribosomal proteins and more than 200 assembly factors and small nucleolar RNAs [4-6]. Abnormalities in the ribosome structure lead to bone marrow failure and physical abnormalities. All ribosomopathies described to date have an increased risk of

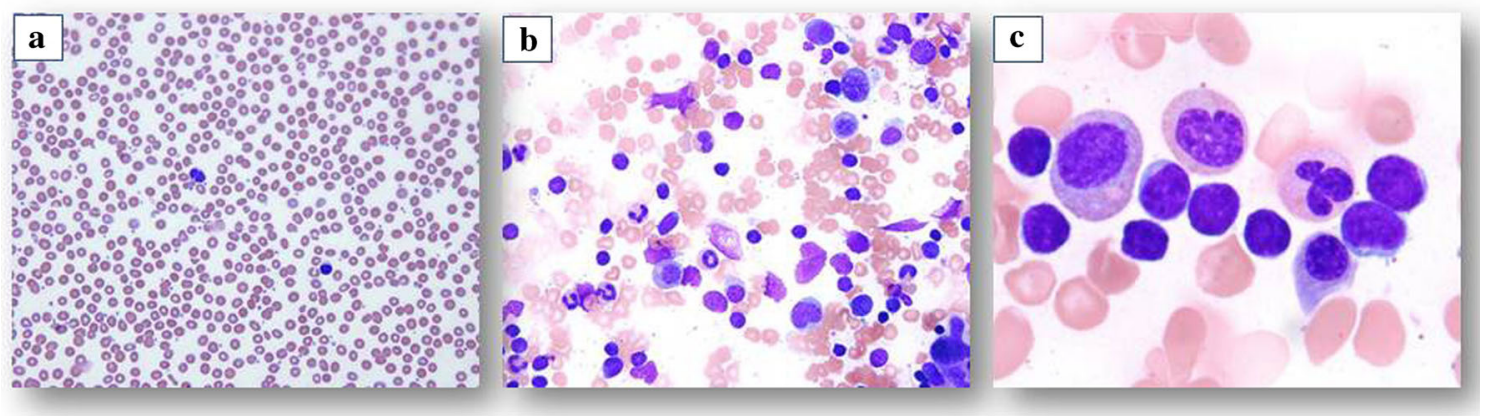

Fig. 1 Diamond Blackfan Anemia. a Peripheral blood, no significant anisopoikilocytosis or polychromasia. b Bone marrow aspirate demonstrating lack of erythroid progenitors and increase number of lymphoid cells. c Immature lymphoid cells consistent with hematogones. (A-C, Wright-Giemsa stain) 
Table 1 Laboratory findings in patients with Diamond-Blackfan anemia

\begin{tabular}{|c|c|}
\hline Laboratory abnormality & Comment \\
\hline $\begin{array}{l}\text { Normochromic macrocytic anemia } \\
\text { Reticulocytopenia }\end{array}$ & $\begin{array}{l}\text { Macrocytosis may be absent during the } 1 \text { st year of life or in patients } \\
\text { with iron deficiency or thalassemia }\end{array}$ \\
\hline $\begin{array}{l}\text { Elevated erythrocyte ademosine } \\
\text { deaminase activity, eADA }(<3 \mathrm{SD})\end{array}$ & $\begin{array}{l}\text { eADA is a weak independent predictor of DBA - it is also elevated } \\
\text { in immune deficiencies, hemolytic anemias, chronic } \\
\text { myeloproliferative disorders, dyskeratosis, or megaloblastic } \\
\text { anemia }\end{array}$ \\
\hline Elevated fetal hemoglobin $(\mathrm{HbF})$ & $\begin{array}{l}\text { Non-specific - also elevated during early infancy, stress erythropoiesis, } \\
\text { in hereditary persistence of hemoglobin F }\end{array}$ \\
\hline Strong expression of $\mathrm{i}$ antigen & Non-specific - also elevated during early infancy, stress erythropoiesis; \\
\hline $\begin{array}{l}\text { Other abnormalities } \\
\uparrow \text { Serum erythropoietin level } \\
\downarrow \text { Red blood cell survival } \\
\downarrow \text { Haptoglobin } \\
\text { Delaied plasma iron clearance } \\
\downarrow \text { Red cell iron utilization }\end{array}$ & Non-specific; \\
\hline
\end{tabular}

malignancy possibly due to the accumulation of free ribosomal proteins that bind to MDM2 and prevent the proteosomal degradation of $\mathrm{p} 53$. The consequent accumulation of p53 leads to cell cycle arrest and apoptosis [7]. In addition, this resultant aberrant expression of p53 can also be of diagnostic value as it does not appear to be increased in acquired causes of anemia [8]. The distinct and overlapping features of ribosomopathies are demonstrated in Fig. 2.

DBA is caused by heterozygous germline mutations in genes encoding key components of the small $40 \mathrm{~S}$ or large $60 \mathrm{~S}$ ribosomal subunits (RPS19 most frequently, RPS24, RPS1, RPS15,
RPS27A, RPS10, RPS29, RPS26, RPL5, RPL11, RPL35A, and $R P L 15)$ [9, 10]. Patients with DBA show reduced colonyforming activity, accelerated apoptosis of BM progenitors, defects in the microenvironment of the BM and altered function of growth factors, their receptors, and transcription factors.

The anemia is usually severe requiring transfusions and the reticulocyte count is markedly decreased with the majority of cases manifesting in the first year of life. Most patients with DBA are treated with steroids and RBC transfusions positing a risk of iron overload with its associated morbidity and mortality. At age 25 , about $20 \%$ of the patients with DBA undergo

Table 2 Causes of acquired pure red cell aplasia in children

\begin{tabular}{|c|c|c|}
\hline Disorder & Pathogenesis & Findings \\
\hline Parvovirus B 19 infection & $\begin{array}{l}\text { Direct infection of early progenitors } \\
\text { Acute transient aplastic crisis in individuals with } \\
\quad \text { impaired red cell production } \\
\text { Chronic anemia in immunosuppressed individuals } \\
\text { Congenital infection }\end{array}$ & $\begin{array}{l}\text { Almost complete absence of erythroid progenitors, } \\
\text { particularly orthochromic erythroblasts } \\
\text { Rare proerythroblasts, occasional giant forms with } \\
\text { inclusion } \\
\text { Characteristic intranuclear inclusions in formalin } \\
\text { fixed tissue }\end{array}$ \\
\hline $\begin{array}{l}\text { Transient erythroblastopenia of } \\
\text { childhood (TEC) }\end{array}$ & $\begin{array}{l}\text { Acquired anemia in previously healthy children } \\
\text { Autoantibodies against red cell progenitors after } \\
\text { infection with unknown virus different from } \\
\text { Parvovirus B19 } \\
\text { Transient, rarely requires bone marrow examination } \\
\text { Excellent prognosis }\end{array}$ & $\begin{array}{l}\text { Temporal reticulocytopenia; rarely leukopenia or } \\
\text { platelet abnormalities } \\
\text { Normal eADA levels } \\
\text { Bone marrow - erythroblastopenia } \\
\text { Increase number of hematogones } \\
\text { Spontaneous recovery is a rule } \\
\text { During recovery phase - marked reticulocytosis } \\
\text { with high MCV }\end{array}$ \\
\hline $\begin{array}{l}\text { Associated with autoimmune } \\
\text { diseases (rare in children) }\end{array}$ & $\begin{array}{l}\text { Rheumatoid arthritis } \\
\text { Systemic lupus erythematosus } \\
\text { As part of a paraneoplastic syndrome } \\
\text { Thymoma, Hodgkin lymphoma }\end{array}$ & Anemia, reticulocytopenia \\
\hline $\begin{array}{l}\text { Drug induced } \\
\text { Phenytoid, Athioprine, Isoniazid }\end{array}$ & $\begin{array}{l}\text { Pathogenesis largely unknown } \\
\text { Direct toxicity? } \\
\text { Immune mediated? }\end{array}$ & $\begin{array}{l}\text { More frequently macrocytosis, rarely cause anemia } \\
\text { Effect reversible after discontinuation of the } \\
\text { offending drug }\end{array}$ \\
\hline
\end{tabular}




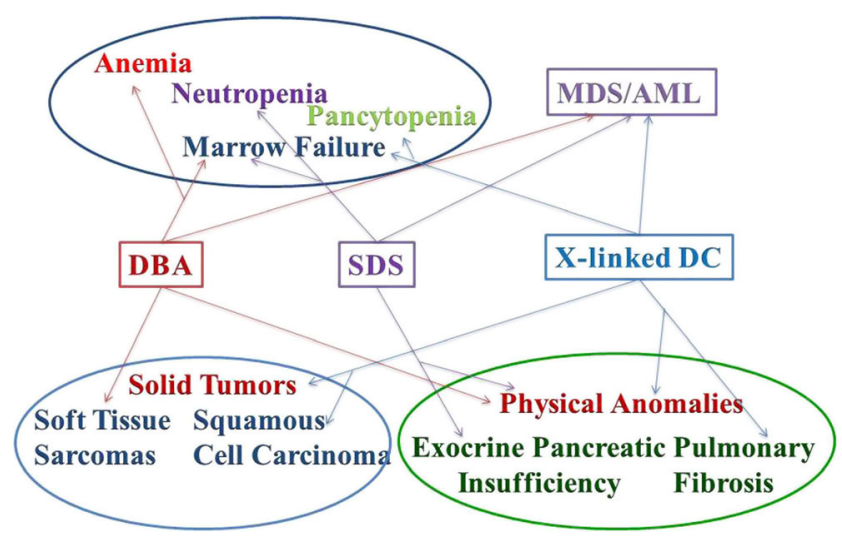

Fig. 2 Schematic representation of the overlapping and distinct features of ribosomopathies

spontaneous remission for reasons that are not well understood. Currently, the only curative option for patients with DBA is allogeneic hematopoietic stem cell transplantation for patients who are refractory to steroids and transfusions.

\section{Congenital dyserythropoietic anemias}

Unlike DBA, where the anemia is due to virtual absence of erythroid progenitors, in CDA, the marrow is hypercellular with erythroid predominance, marked dyserythropoiesis, and accelerated death of erythroid progenitors. As a result, the bone marrow proliferative capacity is markedly impaired and the daily red cell production reduced resulting in marked anemia with reticulocytopenia. Based on the type and degree of dyserythropoiesis, clinical, and serological findings, the CDAs have been classified as classic CDA I, CDA II, CDA III, and CDA variants defined as CDA groups IV to VII [11, 12]. The characteristic findings of the CDAs are summarized in Table 3.

The diagnosis of CDA requires evidence of a hereditary pattern of inheritance, anemia with reticulocytopenia, dyserythropoiesis, and ineffective erythropoiesis. In addition, exclusion of other causes of anemia such as hemoglobinopathies, inherent membrane or enzyme RBC defects, or immune mediated hemolytic anemias is required [13]. While the dyserythropoiesis is a characteristic feature of CDA, it may be minimal in some types of CDA, group IV. Furthermore, dyserythropoiesis is not specific and can be seen in radiation-, drug-, or virally induced dyserythropoiesis, stress erythropoiesis in recovering marrow as well as nutritional deficiencies, myelodysplastic syndrome (MDS), and erythroleukemia. Definitive confirmation of the diagnosis of CDA requires the characteristic clinical presentation, peripheral blood, and bone marrow findings along with typical electron microscopic findings, abnormal band migration on SDS-PAGE, and/or the identification of a characteristic gene mutation.

Unlike DBA, patients with CDA will have clinical and laboratory findings associated with increased red cell destruction (intramedullary) such as jaundice, elevated bilirubin and lactic dehydrogenase (LDH), splenomegaly, iron overload, and other abnormalities findings frequently seen in hemolytic anemias.

The diagnosis of CDA can be challenging because the morphologic findings depend on the type of CDA. In CDA $\mathrm{I}$, the peripheral blood shows characteristic macrocytic anemia with marked anisopoikilocytosis including microcytes and characteristic macroovalocytes, basophilic stippling, and various number of nucleated red blood cells (Fig. 3). The BM aspirate smears show numerous binucleated erythroblasts with asymmetric nuclear lobes and the pathognomonic intranuclear bridges. In addition, megaloblastoid changes and irregular nuclear membrane contours are seen in the remaining erythroid precursors. The probability of CDA I becomes increasingly likely when $20 \%$ or more of the erythroid precursors show the pathognomonic features by light microscopy [14]. Electron microscopy can be helpful and until the advent of reliable genetic testing, was the gold standard for the diagnosis of CDA I. It shows a pathognomonic "Swiss-cheese" heterochromatin pattern indicative of abnormally condensed nuclear material [15]. In addition, nuclear membrane invaginations encompassing cytoplasmic organelles can also be appreciated.

CDA II also known as hereditary erythroblastic multinuclearity with positive acidified serum test (HEMPAS) [16] is the most common type of congenital dyserythropoietic anemia. It is usually clinically apparent in late childhood and early adolescence with normochromic anemia and associated jaundice, splenomegaly, hepatomegaly, and iron overload even in non-transfused patients. As a consequence of the iron overload, approximately $20 \%$ of CDA II patients will develop secondary liver cirrhosis [17].

The peripheral blood smears of patients with CDA II show a characteristically normocytic anemia in a background of anisopoikilocytosis, indirect hyperbilirubinemia, low to absent plasma haptoglobin, and an inappropriately low reticulocyte count [13]. However, presence of macrocytosis or microcytosis does not exclude the diagnosis. Bone marrow aspirates show marked erythroid hyperplasia and dyserythropoiesis with characteristic symmetric binucleation and less frequently multinucleation of the erythroid progenitors. Patients with true CDA II usually show greater than $10 \%$ of erythroid precursors to be binucleated whereas normal individuals have at most $2 \%$ binucleated cells even in periods of erythropoietic demand [14].

Electron microscopy, though no longer routinely performed, shows stretches of double membranes that represent excess smooth endoplasmic reticulum. The historical laboratory test and basis for its original namesake is the acidified serum test (Ham test) in which the defective erythroid precursors will lyse. However, the technical challenges inherent to validating and performing the Ham test have rendered this procedure largely obsolete. Other diagnostic testing methods 
Table 3 Findings of the major subtypes of congenital dyserythropoietic anemias

\begin{tabular}{|c|c|c|c|}
\hline & Type I & Type II (HAMPAS) & Type III, familial \\
\hline Peripheral blood & $\begin{array}{l}\text { Moderate anemia } \\
\text { Macrocytic anemia }(70 \%) \\
\text { Macrocytes present even if } \\
\quad \text { MCV is normal } \\
\text { Marked anisopoikilocytosis } \\
\text { Basophilic stippling } \\
\text { Cabot ring, rare } \\
\text { Rare nucleated RBC }\end{array}$ & $\begin{array}{l}\text { Mild to moderate normochromic anemia } \\
\text { Moderate anisopoikilocytosis } \\
\text { Basophilic stippling } \\
\text { Rare nucleated RBC }\end{array}$ & $\begin{array}{l}\text { Mild macrocytic anemia due to ineffective } \\
\text { erythropoiesis and intravascular hemolysis } \\
\text { Moderate anisopoikilocytosis } \\
\text { Basophilic stippling }\end{array}$ \\
\hline $\begin{array}{l}\text { Bone marrow } \\
\text { findings }\end{array}$ & $\begin{array}{l}\text { Megaloblastoid erythroid } \\
\text { maturation } \\
\text { Polychromatic erythroblasts - } \\
\text { binucleated forms }<20 \% \\
\text { Multiple nuclei have different } \\
\text { size } \\
\text { Intranuclear chromatin } \\
\text { bridges, rare }\end{array}$ & $\begin{array}{l}\text { Normoblastic maturation } \\
\text { Binucleated forms } 10-45 \% \text {, orthochromic } \\
\quad \text { erythroblasts } \\
\text { Binucleated cells have same size nuclei }\end{array}$ & $\begin{array}{l}\text { Giant multinucleated erythroblasts with up to } \\
12 \text { nuclei per cell and DNA content up to } \\
14 \mathrm{~N}^{\mathrm{a}} \\
\text { The individual nuclei of some multinucleated } \\
\text { cells vary in size and DNA content }\end{array}$ \\
\hline $\begin{array}{l}\text { Ultrastructural } \\
\text { studies }\end{array}$ & $\begin{array}{l}\text { Spongy, "Swiss-cheese", } \\
\text { chromatin } \\
\text { Typical binucleated forms } \\
\text { Intranuclear chromatin bridges } \\
\text { Condensation of the chromatin } \\
\text { indicative of apoptosis } \\
\text { Iron loaded mitochondria }\end{array}$ & $\begin{array}{l}\text { Peripheral double membrane due to } \\
\text { excess of rough endoplasmic } \\
\text { reticulum }\end{array}$ & $\begin{array}{l}\text { Non-specific - intranuclear clefts and } \\
\text { karyorrhexis }\end{array}$ \\
\hline $\begin{array}{l}\text { Laboratory } \\
\quad \text { abnormalities }\end{array}$ & $\begin{array}{l}\text { Ham test negative } \\
\text { High bilirubin } \\
\text { Increased ferritin } \\
\text { Decrease glycosilation }\end{array}$ & $\begin{array}{l}\text { Ham test positive, characteristic } \\
\text { High bilirubin } \\
\text { Increased ferritin } \\
\text { Impaired globin chain synthesis } \\
\text { Defective glycosilation }\end{array}$ & $\begin{array}{l}\text { Ham test negative } \\
\text { Slightly increased bilirubin } \\
\text { Normal iron, ferritin, and transferrin } \\
\text { Evidence of intravascular hemolysis: } \\
\text { Decreased or absent haptoglobin, } \\
\text { Markedly elevated LDH } \\
\text { Hemosidenuria }\end{array}$ \\
\hline Other abnormalities & $\begin{array}{l}\text { Iron overload } \\
\text { Gallstones } \\
80-90 \% \text { splenomegaly } \\
\text { Congenital anomalies }\end{array}$ & $\begin{array}{l}\text { Iron overload } \\
\quad 20 \% \text { develop liver cirrhosis } \\
\text { Gallstones } \\
\text { Splenomegaly } \\
\text { Congenital anomalies, infrequent }\end{array}$ & $\begin{array}{l}\text { No } \\
\text { High incidence of lymphoproliferative } \\
\quad \text { disorders }\end{array}$ \\
\hline Inheritance & Autosomal recessive & $\begin{array}{l}\text { Autosomal recessive } \\
\text { South Italy, founder effect }\end{array}$ & $\begin{array}{l}\text { Autosomal dominant } \\
\text { Several families in Northern Sweden, } \\
\text { North America, and Argentina } \\
\text { Autosomal recessive in sporadic forms }\end{array}$ \\
\hline
\end{tabular}

${ }^{\mathrm{a}} N=$ haploid DNA content

include sodium dodecyl sulfate polyacrylamide gel electrophoresis (SDS-PAGE) which is sensitive and specific for CDA II which shows narrower banding and faster migration of the band-3 and band- 4.5 proteins secondary to abnormal glycosylation [18] and targeted genetic testing.

The rarest of all of the CDAs, CDA III was originally described and characterized in 1951 in the USA [19] and in 1962 in two Swedish patients with thorough evaluation of familial records as far back as the mid-eighteenth century [20]. CDA III shows an autosomal dominant pattern of inheritance and unlike CDA I and II, there is a slight increase in the bilirubin with normal iron, ferritin, and transferrin in addition to evidence of intravascular hemolysis such as decreased or absent haptoglobin, elevated LDH, and hemosiderinuria. Because of the rarity of this disorder, the associated non- erythropoietic sequelae have only been described to a limited extent. The only reproducible non-erythropoietic associations with CDA III are ocular abnormalities and multiple myeloma within their respective familial cohorts.

Definitive evaluation for the disorder requires a bone marrow biopsy where the pathognomonic abundant "giant" erythroid precursors at all levels of maturation can be appreciated. While multinucleation is a recurrent feature of all the CDAs, CDA III shows far greater multinucleation with up to $12 \mathrm{nu}$ clei per cell, a unique feature for this disorder.

\section{CDA, variants}

There are several CDA variants that do not fulfill the morphologic or biochemical criteria for the diagnosis of CDA I-III. 
Fig. 3 Congenital dyserythropoietic anemia type I. a Peripheral blood, marked anisopoikilocytosis, macroovalocytes, and basophilic stippling (Wright-Giemsa stain). b Bone marrow aspirate, marked erythroid hyperplasia with dyserythropoiesis including internuclear bridge and uneven size of the dysplastic nuclear lobes (Wright-Giemsa stain). c Bone marrow biopsy, hypercellular marrow with marked erythroid hyperplasia (H\&E). d Increased number of sideroblasts and storage iron (Iron stain)
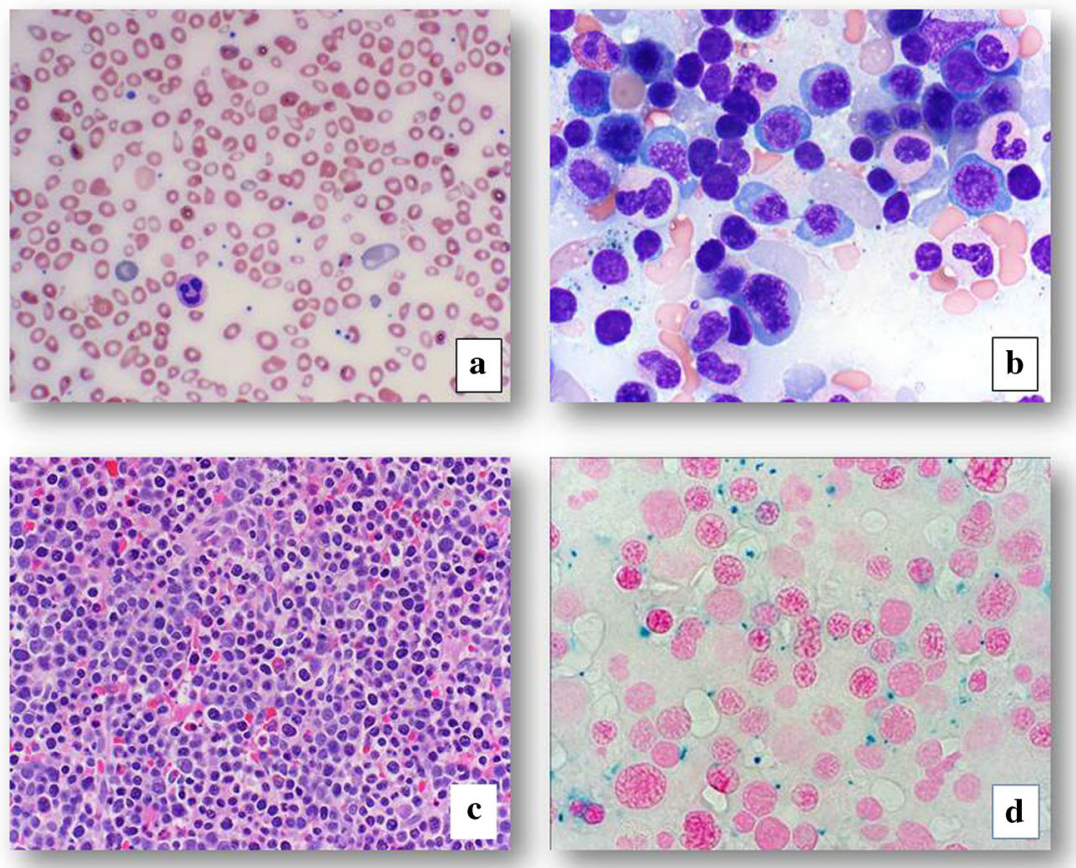

Based on light microscopy and EM of the bone marrow, four additional groups have been classified as CDA IV-VII [12]. Furthermore, congenital dyserythropoietic anemia can be part of a broader syndrome such as Majeed syndrome, CDA with exocrine pancreatic insufficiency, and mevalonate kinase deficiency [21].

\section{Genetics of CDA}

CDA I is inherited in autosomal recessive (AR) or autosomal dominant (AR) mode. The underlying genetic defect of the AR form has been linked to the gene $C D A N-1$ that encodes the protein codanin-1 [22]. Codanin-1 is a ubiquitously expressed protein, and recent studies demonstrated that it binds to Asfla, a histone chaperone, and regulates a limited step in chromatin replication [23]. It is still not completely understood how such a defect would lead to the phenotype of CDA I. Recently, whole genome sequencing of a Pakistani patient identified a new CDA I gene, C150RF41 [21]. However, the function of this protein is not fully understood.

CDA II is also an autosomal recessive disorder, and mutations in the secretory pathway gene $S E C 23 B$ encoding the protein COPII have been identified [24]. Further studies have shown that defects of COPII directly result in the binucleation of erythroid precursors.

Until recently, the underlying genetic aberration for CDA III was unknown largely because of its rarity. However, the genetic defect has been linked to the gene KIF23 on chromosome 20 located telomeric to the CDA II locus. The gene product, mitotic kinesin-like protein 1, plays a critical role in cytokinesis.
Mutations in erythroid transcription factors GATA1 and $K L F 1$ have been demonstrated in patients with CDA variants. GATA1, a transcription factor with a key role in the erythropoiesis and megakaryopoiesis, mutations have been identified in a few documented familial inheritance forms of X-linked thrombocytopenia characterized by macrothrombocytopenia, bleeding diathesis, and dyserythropoiesis leading to a various degree of anemia from hydrops fetalis to asymptomatic forms [21]. Heterozygote dominant negative mutations of KLF1 have been identified in patients with CDA group IV [25]. Such patients have autosomal dominant mode of inheritance. It is important to note that $K L F 1$ mutations leading to loss of function are also identified in several benign hematologic conditions such as familial persistence of hemoglobin F, borderline elevation of hemoglobin A2, and others [26].

\section{Current diagnostic approach to CDA}

When the clinical presentation, laboratory findings, peripheral blood, and bone marrow changes are suggestive of CDA type I to III and other causes of dyspoiesis are excluded, the diagnosis is confirmed by sequencing of appropriate genes. For such patients, there is no need to perform additional specialized testing such as BM electron microscopy or SDS-PAGE. A CDA variant should be suspected for patients with clinical and laboratory findings compatible with CDA but show absence of bone marrow morphologic findings consistent with CDA I to III. For these patients, DNA sequencing and mutations in KLF1 and GATA1 should be considered. For patients with no identifiable mutations, electron microscopy and SDSPAGE can be helpful to confirm the diagnosis. 


\section{Bone marrow failure syndromes associated with neutropenia}

\section{Shwachman-Diamond syndrome}

Shwachman-Diamond syndrome (SDS) is a ribosomopathy characterized by neutropenia, associated pancreatic exocrine function insufficiency, and metaphyseal dysostosis [27]. Most patients present early in life with malabsorption due to pancreatic insufficiency and recurrent infections. In addition, patients with SDS have a range of physical findings such as short stature, failure to thrive, skin rashes, teeth and palatal defects, and syndactyly. However, some patients may present insidiously later in life.

Neutropenia is the most common hematologic abnormality and although seen in all patients, it is constant in only a third of them and intermittent in the rest [28]. In addition, all patients have impaired neutrophil chemotaxis. Other hematologic abnormalities include anemia, seen in two-thirds of the patients, and intermittent thrombocytopenia seen in a third of the patients. Unlike DBA and CDA, the anemia in SDS is associated with an appropriate bone marrow response and an increased reticulocyte count. A majority of patients present with elevated hemoglobin F levels.

The bone marrow findings are overall non-specific. The marrow cellularity varies, and while some patients are hypocellular, others show normocellular or hypercellular marrow. The overall cellularity of the marrow may be increased due to the increased number of normal B cell progenitors, hematogones (Figs. 4 and 5). However, this is not unique to SDS and can be seen in other IBMF syndromes.

Individuals with SDS have a high risk of overall bone marrow aplasia, myelodysplastic syndrome, and predisposition to acute myeloid leukemia. SDS is associated with increasing benign clonal cytogenetic abnormalities such as i(7)(q10) and $\operatorname{del}(20)(\mathrm{q} 11)$. These abnormalities may persist for years and alone do not appear to be sufficient for neoplastic transformation and subsequent development of MDS and AML. Of note, SDS patients are at an increased risk of treatment complications and may need dose adjustments of chemotherapy. Thus, establishing a proper diagnosis is essential for the proper management of these patients.

\section{Genetics of SDS}

SDS is inherited as an autosomal recessive disorder. Mutations at a single gene, $S D B S$, are identified in significant number of the patients with SDS, but not in all. The SDBS gene plays an important role in RNA metabolism and ribosomal function such as regulation of the large $60 \mathrm{~S}$ ribosomal subunit and its subsequent joining to the smaller $40 \mathrm{~S}$ subunit to form the mature ribosome. In addition, the $S D B S$ gene product has important functions in mitotic spindle stabilization and chemotaxis. These findings suggest that the SDS phenotype facilitates the genomic instability. However, the nonribosomal functions of $S D B S$ gene, such as the mitotic spindle stabilization, may play a more important role in the progression to malignancy [27]. The lack of mutations in some patients with SDS suggests additional genes may play role in the pathogenesis of this disorder.

\section{Current diagnostic approach to SDS}

The diagnosis in SDS can be difficult. The presence of neutropenia with characteristic exocrine pancreatic insufficiency should prompt sequencing of the SDBS gene. However, the lack of a mutation at this gene does not exclude SDS. For children that present with peripheral cytopenias and increased number of immature $\mathrm{B}$ cells in the marrow, demonstration of a maturation pattern of the $\mathrm{B}$ cells is pivotal in avoiding misdiagnosis of B lymphoblastic leukemia. The differential diagnosis of SDS also includes other IBMF syndromes associated with failure of myelopoiesis and isolated neutropenia such as the severe congenital neutropenia syndromes (SCN). Fanconi anemia and dyskeratosis congenita have to be excluded in patients that present with pancytopenia and bone marrow aplasia.

\section{Severe congenital neutropenia}

Severe congenital neutropenia ( $\mathrm{SCN})$ is a genetically and phenotypically heterogeneous disorder that presents early in infancy with profound neutropenia and infections. The WBC can be normal or decreased with severe neutropenia, below $0.5 \times 10[9] / \mathrm{L}$, and frequently associated monocytosis and eosinophilia. While some patients with SCN have persistent neutropenia and severe life-threatening infections, others present with cyclic fluctuations of the neutrophil count with a classic 21-day periodicity (cyclic neutropenia).

In $\mathrm{SCN}$, the bone marrow is normocellular and shows absence of mature granulocytes with maturation. Those granulocytes that are present are arrested at the promyelocyte or myelocyte stages. Erythropoiesis and megakaryopoiesis are preserved, and the number of immature B cell progenitors is usually increased.

$\mathrm{SCN}$ is genetically heterogeneous and can arise from mutations affecting multiple pathophysiologic pathways all ultimately leading to apoptosis of marrow progenitors. Heterozygous mutations at ELA-2/ELANE are the most frequent and are found in about $50 \%$ of patients with severe congenital neutropenia inherited as autosomal dominant or sporadic forms [29]. ELA-2 encodes the enzyme neutrophil elastase expressed in neutrophils and to a lesser degree in monocytes. Mutations at $E L A-2$ result in protein misfolding leading to apoptosis. The original autosomal recessive form of SCN first 
Fig. 4 Shwachman-Diamond syndrome. a Hypercellular bone marrow with increase number of small cells diffusely infiltrating the marrow (H\&E). b CD20 immunohistochemical stain demonstrating a small number of mature B lymphocytes. c PAX5 immunohistochemical stain demonstrating a much higher number of B lymphocytes. Of note, PAX5 is expressed very early in B cell maturation and will be positive in both immature and mature B cells. d TdT

immunohistochemical stain demonstrating scattered immature cells. This maturation pattern is consistent with normal B cells, hematogones
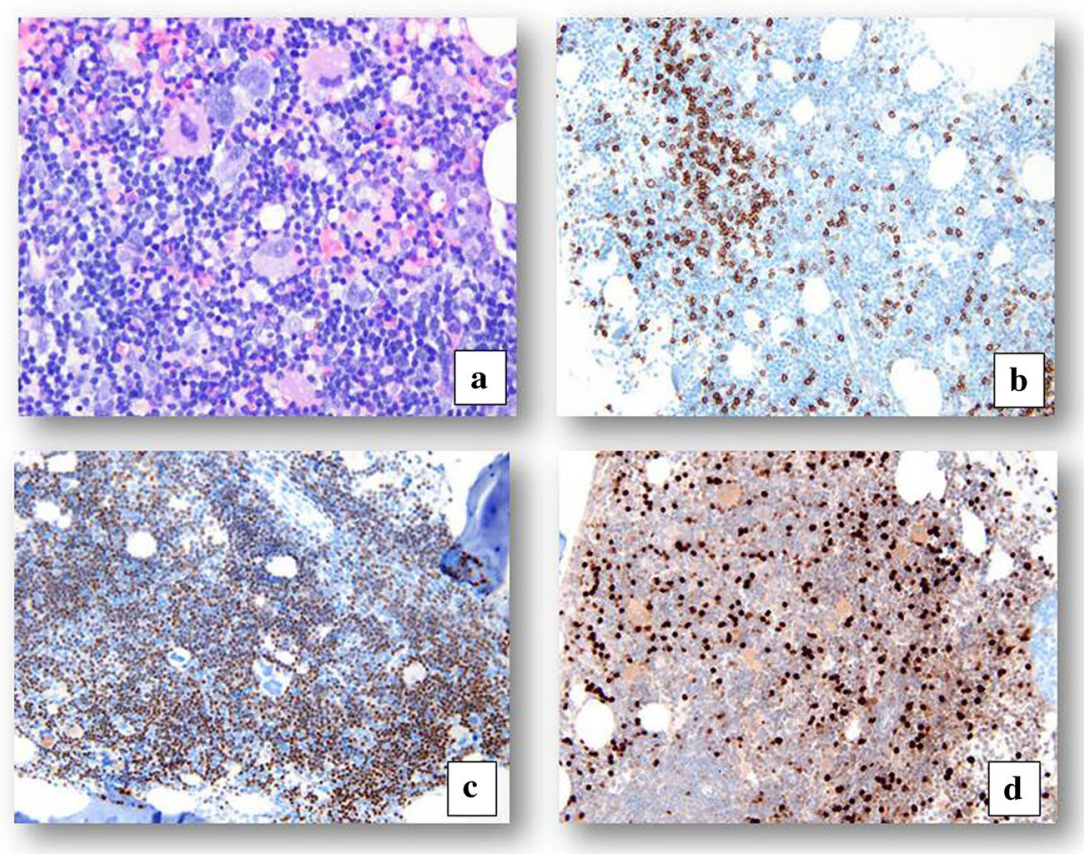

described by Kostmann is due to mutations at $H A X 1$, a mitochondrial gene with homology to the BCL2 family, resulting in a pro-apoptotic state. Mutations at GFI, G6PC3,WAS, and $C S F 3 R$ genes also have been reported.
Patients with SCN have a high risk of developing MDS/ AML. In addition, a subset of patients with SCN may develop subsequent acquired somatic mutations to the granulocyte colony stimulating factor receptor (G-CSFR) $C S F 3 R$ leading to
Fig. 5 Multiparameter flow cytometry demonstrating an increased number of normal B cell progenitors demonstrating characteristic maturation pattern showing variable expression of CD34, CD20, and CD38
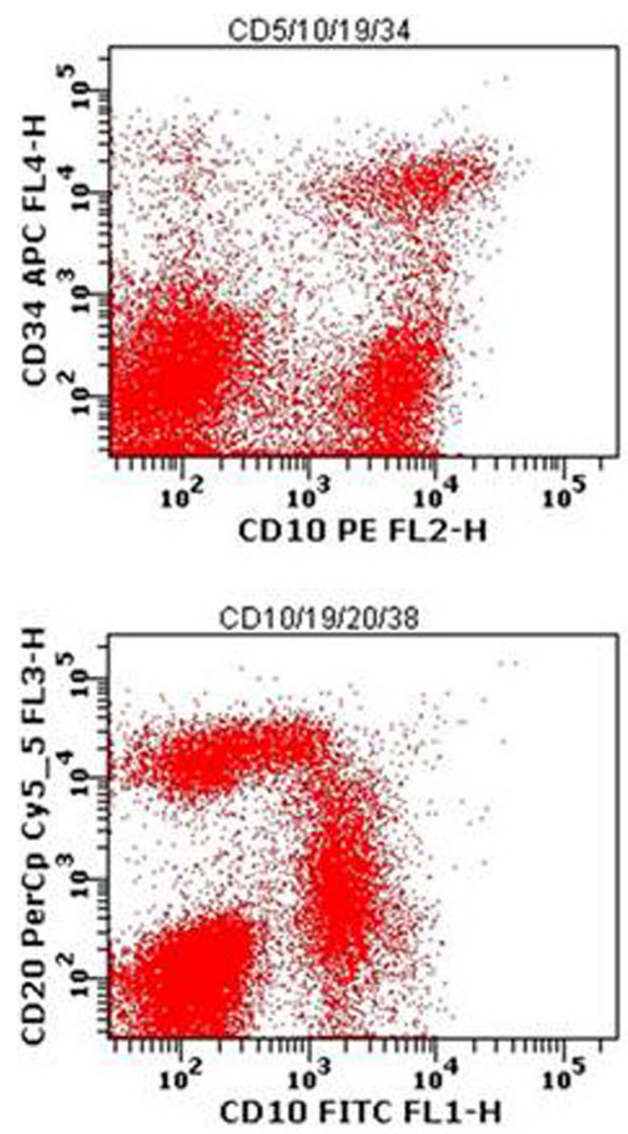
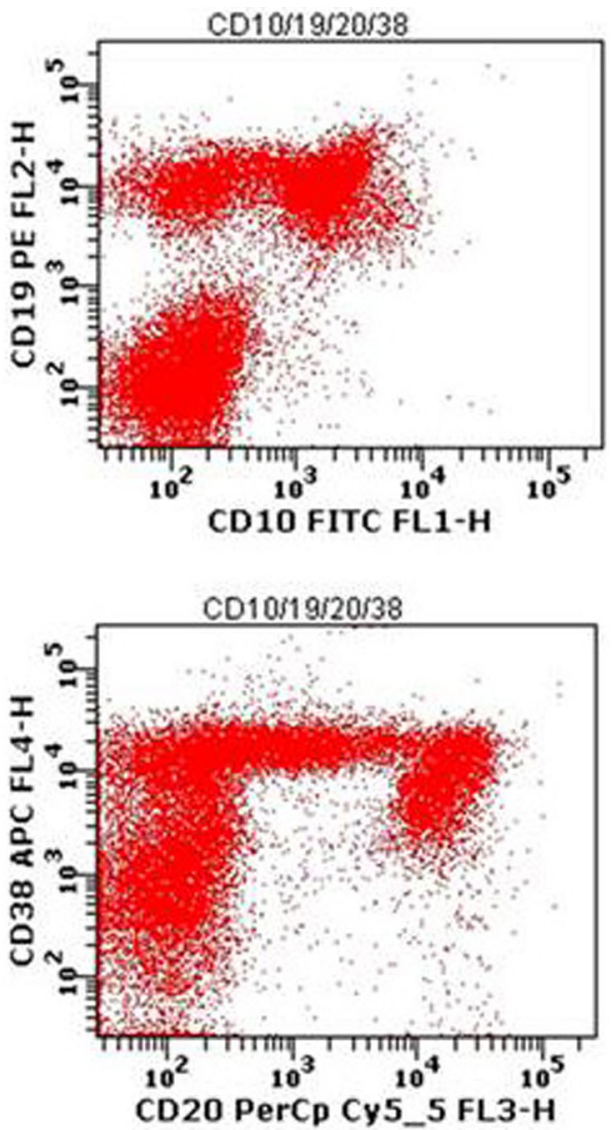
expression of truncated CSF3R protein. Cells harboring such mutations have increased proliferative capacity and resistance to apoptosis.

Prior to G-CSF, patients with SCN had a dismal prognosis. Therapy with G-CSF results in an increase in the absolute neutrophil count $(>1.5 \times 10[9] / \mathrm{L})$ and decreases the rate and severity of infections. However, the role of G-CSF therapy in the cancer predisposition is still controversial. Despite patients treated with G-CSF having a higher risk to develop MDS/ AML as compared to patients without growth factor therapy, a baseline risk none the less remains. The risk of MDS/AML is the highest however for patients with a poor G-CSF response requiring high doses of G-CSF therapy.

The diagnosis of SCN is based on the persistent severe neutropenia frequently associated with monocytosis and eosinophilia in the peripheral blood and normocellular bone marrow demonstrating granulocytic lineage maturation arrest at promyelocyte/myelocyte stage. In patients on G-CSF, the myeloid progenitors show variable degrees of maturation as well as dyspoiesis including prominent granulation. Acquired partial or complete loss of chromosome 7 and abnormalities of chromosome 21 can occur in association with MDS and can be seen in patients treated with G-CSF. The differential diagnosis includes other causes of neutropenia in newborns and infancy such as infections, drug-induced neutropenia, alloimmune neutropenia, metabolic disorders, and congenital immunodeficiency syndromes (Table 4). Immune mediated neutropenia due to anti-neutrophil antibodies should also be considered, and in such cases, the bone marrow shows complete maturation of the myeloid progenitors to band stage.

\section{Bone marrow failure syndromes associated with multiple cytopenias}

\section{Fanconi anemia}

Fanconi anemia (FA) is the most frequent cause of bone marrow failure characterized by physical anomalies, progressive bone marrow failure, and cancer predisposition [29]. FA is a genetically heterogeneous disease defined by complementation groups. Currently, germline mutation in one of 16 genes has been identified leading to defective DNA damage response [9]. The current diagnostic criteria rely on identifying increased chromosomal breakage in cells cultured in DNA-crosslinking agents.

The manifestation of FA depends on the patient's age. Early in life, the physical abnormalities are the predominant feature. A majority of patients, $60 \%$, have at least one physical anomaly including short stature, hypo- and hyperpigmented skin lesions, café au lait spots, and abnormalities of the radial ray $(30 \%)$, all involving the thumb. Microcephaly, microphthalmia, structural renal abnormalities, and hypogonadism are present in 20-25\% of the cases. About
2/3 have endocrine abnormalities including short stature and growth hormone deficiency, hypothyroidism, midline brain abnormalities, abnormal glucose/insulin metabolism, obesity, and dyslipidemia. In addition, a majority of patients with FA develop bone marrow failure between ages 5 and 15 years with a median age of diagnosis of 6.5 years.

The most common hematologic abnormalities in FA are pancytopenia with macrocytosis and persistence of fetal hemoglobin. Thrombocytopenia usually precedes the anemia and neutropenia. The bone marrow findings range from normal cellularity to complete marrow aplasia with increased numbers of lymphocytes, plasma cells, and mast cells to full aplasia indistinguishable from idiopathic aplastic anemia. Various degrees of dyshematopoiesis may also be present. Many patients with FA have cytogenetic clones such as monosomy 7 or add $3 \mathrm{q}$. While the prognostic significance of such clones is unclear, the expansion of clones with proliferative advantages does play a role in development of MDS/AML [30]. For the diagnosis of MDS in the setting of FA, the presence of peripheral cytopenias as well as degree of dyshematopoiesis in the bone marrow are more important features than the presence of a cytogenetic clone alone. Complications leading to death include sepsis or bleeding in patients with aplastic anemia, complications of stem cell transplantation, and cancers.

There is a correlation between the extent of physical anomalies and hematologic presentation with the risk of acute leukemia and solid tumors. While patients with many birth defects are more likely to develop BM failure, the patients with a normal physical appearance have a higher risk of developing acute leukemia and solid tumors as young adults. The most common malignancies include AML, head and neck squamous cell carcinoma (HNSCC), liver tumors, vaginal squamous cell carcinoma, and brain tumors. The relative risk of developing AML is more than 600-fold and for HNSCC is about 500-fold higher as compared to the general population. One specific genotype (FANCD1/BRCA2) has a high rate of birth defects and the highest risk of cancer as by age 6, more than $95 \%$ develop midline brain tumors, Wilms tumors, or AML. In addition, the relative risk of MDS is 5000-fold higher as compared to the general population.

\section{Genetics of FA}

FA is due to a germline mutation resulting in a defective DNA damage response. Except for FANCB mutations that are Xlinked, all other mutations are inherited as an autosomal recessive disease. At least $95 \%$ of patients have germline mutations in one of 16 genes encoding proteins involved in the common DNA repair pathway [9]. Additional functions include stress signaling and apoptosis in response to oxidative damage and inflammatory cytokines.

As a result of abnormal DNA repair, the cells of FA are uniquely hypersensitive to the clastogenic effect of DNA- 
Table 4 Causes of acquired neutropenia in children

\begin{tabular}{ll}
\hline Disorders & Characteristic features \\
\hline Neonatal alloimmune neutropenia & Neutrophil specific maternal IgG due to maternal alloimmunization \\
& Affected infants may be asymptomatic \\
Autoimmune neutropenia & Primary, most frequent in infants and young children \\
& Secondary, as a part of autoimmune disorder (hemolytic anemia, immune mediated thrombocytopenia, \\
& Evans syndrome) \\
Antineutrophil antibodies, presumed peripheral destruction of antibody coated neutrophils \\
Most common classes \\
Antimicrobials: Trimethoprim/sulfamethozasole , Penicillins, Chloramphenicol \\
Anticonvulsant: valproic acid \\
Anti-thyroid: Propylthiouracil \\
Anti-psychotics: Clozapine \\
Idiosyncratic reactions, immune mediated destruction, or direct toxicity \\
Viral: EBV, hepatitis A and B, HIV, influenza A and B, measles, RSV, parvovirus B19, \\
Infection & rubella, varicella) \\
& Bacterial: sepsis \\
Nutritional deficiencies & Bone marrow - megaloblastoid maturation \\
Reticuloendothelial sequestration & Hypersplenism due to portal hypertension \\
Bone marrow infiltration & Moderate neutropenia \\
Chronic idiopathic neutropenia & Bone marrow involvement by leukemia, lymphoma or solid tumors \\
& Granulomatous infections, lysosomal storage diseases, osteopetrosis \\
& Poorly understood disorders with variable clinical features, bone marrow findings, and clinical history
\end{tabular}

${ }^{\text {a }}$ Italicized are the drugs with highest relative risk of causing neutropenia

crosslinking agents such as mitomycin $\mathrm{C}$ and diepoxybutane (DEB) and show increase breakage. FA cells exposed to DEB show a significantly higher number of chromosome breaks as compared to a normal control. This test is unique to the A phenotype and can be used as an initial screening test. For the majority of patients, the diagnosis of FA can be made with careful clinical history, physical findings, and chromosome breakage studies. However, about $30 \%$ of FA may present with no significant physical abnormalities. Therefore, the DEB test is very helpful and should be systemically used to screen patients with aplastic anemia.

\section{Dyskeratosis congenita}

The classical clinical presentation of dyskeratosis congenita (DC) is characterized by mucocutaneous abnormalities (i.e., abnormal skin pigmentation, nail dystrophy, and leukoplakia), bone marrow failure, and a predisposition to malignancies. However, abnormalities of every organ system have been described owing to the underlying genetic disorder (described below). The classic cutaneous abnormalities appear before the age of 10 years. Bone marrow failure is the principle cause of mortality with approximately $80 \%$ of patients showing signs before the age of 30 years. Initially, while only one hematopoietic lineage may be affected, over time pancytopenia develops. The bone marrow shows variable changes from normal cellularity to full-blown aplasia similarly to idiopathic aplastic anemia. The diagnosis can be particularly challenging if no physical stigmata of DC are present.

The most common complications of DC are pulmonary and vascular. In addition, such patients have an increased risk of developing MDS and AML. The only long-term cure for the hematopoietic abnormalities is allogeneic hematopoietic stem cell transplantation. Unfortunately, DC patients have a higher than average risk of mortality associated with stem cell transplantation most likely secondary to the underlying systemic telomere defect.

DC is inherited as X-linked, autosomal dominant, and autosomal recessive forms. De novo mutations are also relatively frequent and currently at least $70 \%$ of individuals with DC have identifiable mutations in one of the telomere maintenance genes (DKC1, TERC, TERT, TINF2, NHP2, NOP10, WRAP53 (protein TCAB1), CTC1, and RTEL1). The most common mode of inheritance, $\mathrm{X}$-linked recessive, is associated with the $\mathrm{DKCl}$ gene encoding a core component of telomerase protein dyskerin.

As a consequence of abnormal telomere maintenance, the telomeres of individuals with DC are markedly shorter, below the 1st percentile [31]. Thus, determining the telomere length is a helpful diagnostic test for DC. DC shows a diverse clinical presentation as the same mutation can present differently with respect to severity, abnormalities, or age at presentation. In some patients with DC, the bone marrow failure may precede the manifestation of non-hematologic abnormalities. Various studies show that up to $5 \%$ of patients with severe idiopathic aplastic anemia (AA) carry a mutation in one of the telomere 
genes associated with DC phenotype [10]. Thus, regular screening of telomere length can identify patients with DC and is important prior to initiation of therapy of AA patients.

Three methods are currently used for measuring of the telomere length: Southern blot, real time polymerase chain reaction, and flow cytometry and fluorescence in situ hybridization (flow-FISH). Of those, flow-FISH has been shown to be the most beneficial in screening patients with aplastic anemia. It should be noted that short telomeres are also seen in other non-DC IBMF syndromes such as DBA, SDS, and FA. However, in contrast to DC patients where the telomere length is below the 1st percentile found in normal controls, the nonDC patients show telomere length clusters in the low normal range [31]. Measuring the telomere length in family members of DC patients is important to identify silent carriers as well as candidates for hematopoietic stem cell donors.

\section{Other less common bone marrow failure syndromes}

\section{Congenital amegakaryocytic thrombocytopenia}

The congenital amegakaryocytic thrombocytopenias (CAMT) are very rare with less than 100 reported cases in the medical literature. Clinically, patients present initially with isolated moderate to severe thrombocytopenia in the absence of physical anomalies. However, there is heterogeneity in the degree of thrombocytopenia , and more severe manifestations of the disorder will subsequently affect all hematopoietic lineages. Patients with CAMT can progress to bone marrow aplasia and have an increased risk of progression to MDS/AML.

All of the CAMT disorders are secondary to biallelic mutations in MPL gene encoding the thrombopoietin (TPO) receptor. Depending on the nature of the underlying defects, CAMT has been divided into three types. Type I CAMT, the most severe manifestation characterized by severe and early onset thrombocytopenia, is caused by nonsense mutations within the intercellular domain of the TPO receptor. Type II CAMT shows a clinically milder and later presentation than type I CAMT and is secondary to missense mutations within the extracellular domain of the TPO receptor. Type III CAMT is defined by ineffective megakaryopoiesis with no discernible defects in the MPL gene.

In the peripheral blood, platelets are of normal size in the background of an otherwise normal appearing smeared and white blood cells. Serial bone marrow biopsies may be required to fully appreciate the absolute decrease of megakaryocytes in type I CAMT as the initial findings may be relatively subtle. TPO levels have also been shown to be of diagnostic utility. TPO is manufactured by the liver at a constant rate and largely removed from the circulation by megakaryocytic receptor mediated uptake and subsequent destruction. In severe manifestations of CAMT, TPO levels can show a 10fold increase from normal limits.
The long-term prognosis of type I and type II CAMT is dismal as most reported cases progress to overall bone marrow failure through unknown mechanisms. In addition, these patients are at an increased risk for myelodysplasia and acute myeloid leukemia. The only curative therapy described to date is hematopoietic stem cell transplant.

\section{Wiskott-Aldrich syndrome}

Wiskott-Aldrich syndrome is a rare $\mathrm{X}$-linked primary immunodeficiency syndrome characterized by microthrombocytopenia in association with eczema, recurrent infections, and increased incidences of autoimmunity and malignancy. For the purposes of this discussion, only the peripheral blood and bone marrow findings of Wiskott-Aldrich will be discussed.

The peripheral blood smears of patients affected by Wiskott-Aldrich syndrome show the classically associated small platelets. Quantification of the mean platelet volume shows the average platelet volume of WAS patients to be, on average, half that of normal. The bone marrows of patients with WAS show overall normal megakaryocyte morphology in a background of an otherwise normal bone marrow.

Genetic studies have shown the WASP gene that is exclusively expressed in hematopoietic cells and functions as a multidomain protein necessary to relay signals from the cell surface to the actin cytoskeleton to be responsible for the clinical phenotype. In addition, a direct correlation exists between the level of the WASP protein and the severity of the clinical phenotype; decreased levels of WASP protein result in a more severe clinical phenotype. Mild mutations in WASP result in X-linked thrombocytopenia.

Diagnosis of the congenital thrombocytopenias requires exclusion of various acquired causes of thrombocytopenia. Isolated thrombocytopenia in infancy and young children is almost always attributable to an immune mediated response within a clinically appropriate context. The peripheral blood smear of patients with increased peripheral destruction/ sequestration of platelets often show morphologically large/ giant megakaryocytes with a concomitant increased mean platelet volume unlike those of true CAMT or WAS where normal size or small platelets are characteristic. Also in contrast to CAMT, all causes of peripheral destruction of platelets on bone marrow biopsy will show normal to increased morphologically unremarkable megakaryocytes.

\section{Work up of a child with suspected bone marrow failure syndrome}

Early recognition and proper diagnosis of IBMF syndromes is important since in addition to the hematologic abnormalities associated with bone marrow failure, many of these patients have a high risk of developing hematologic and nonhematologic malignancies. The workup of a child with a 
suspected inherited bone marrow failure syndrome can be challenging and requires a comprehensive approach and correlation of the clinical history, presence of physical abnormalities, age of hematologic presentation, type and degree of cytopenia, and the peripheral blood and bone marrow findings. Additional studies such as cytogenetics, DEB chromosome breakage studies, flow cytometry, telomere length measurement, and others may be necessary. Knowledge on the indications and limitations for various laboratory tests are essential for their proper utilization. For example, DEB is sensitive and specific for the diagnosis of FA and should be included in the work up of a child with suspected FA as well as in aplastic anemia since many patients with FA do not have the physical stigmata or history of FA. The telomere length is below the 1st percentile in patients with DC but can be decreased in other IBMF syndromes, thus confirming the clinical suspicion.

Next generation sequencing is becoming a powerful technique in the diagnosis of IBMF syndromes as well as a tool for providing new insights of the pathogenesis of these disorders. Currently, there are no well-defined indications for such studies, and the clinical relevance of some of the findings is still not determined. However, the utility of this technique, coupled with its ever decreasing cost, will expand.

\section{References}

1. Vlachos A, Ball SE, Dahl N et al (2008) Diagnosing and treating Diamond Blackfan anaemia: results of an international clinical consensus conference. Br J Haematol 142:859-876. doi:10.1111/j.3652141.008.07269.x, Epub 2008 Jul 30

2. Vlachos A, Rosenberg PS, Atsidaftos E, Alter BP, Lipton JM (2012) Incidence of neoplasia in Diamond Blackfan anemia: a report from the Diamond Blackfan Anemia Registry. Blood 119: 3815-3819. doi:10.1182/blood-2011-08-375972, Epub 2012 Feb 23

3. Chirnomas S, Kupfer G (2013) The inherited bone marrow failure syndromes. Pediatr Clin North Am 60:1291-1310. doi:10.016/j. pcl.2013.09.007

4. Ruggero D, Shimamura A (2014) Marrow failure: a window into ribosome biology. Blood 124:2784-2792

5. Ganapathi KA, Shimamura A (2008) Ribosomal dysfunction and inherited marrow failure. Br J Haematol 141:376-387

6. Narla A, Ebert BL (2010) Ribosomopathies: human disorders of ribosome dysfunction. Blood 115(16):3196-3205

7. Khanna-Gupta A (2013) Bone marrow failure syndromes: The ribosomopathies. Journal of bone marrow research 1:11752

8. Elghetany MT, Alter BP (2002) p53 protein overexpression in bone marrow biopsies of patients with Shwachman-Diamond syndrome has a prevalence similar to that of patients with refractory anemia. Arch Pathol Lab Med 126:452-455

9. Khincha P, Savage S (2013) Genomic characterization of the inherited bone marrow failure syndromes. Semin Hematol 50: 333-347. doi:10.1053/j.seminhematol.2013.09.002

10. Sakaguchi H, Nakanishi K, Kojima S (2013) Inherited bone marrow failure syndromes in 2012. Int J Hematol 97:20-29
11. Heimpel H, Wendt F, Klemm D, Schubothe H, Heilmeyer L (1968) Congenital dyserythropoietic anemia. Arch Klin Med 215:174-194

12. Wickramasinghe SN, Wood WG (2005) Advances in the understanding of the congenital dyserythropoietic anaemias. Br J Haematol 131:431-446

13. Heimpel H (2004) Congenital dyserythropoietic anemias: epidemiology, clinical significance, and progress in understanding their pathogenesis. Ann Hematol 83:613-621

14. Heimpel H, Kellermann K, Neuschwander N, Högel J, Schwarz K (2010) The morphological diagnosis of congenital dyserythropoietic anemia: results of a quantitative analysis of peripheral blood and bone marrow cells. Haematologica 95:1034-1036

15. Heimpel H, Forteza-Vila J, Queisser W, Spiertz E (1971) Electron and light microscopic study of the erythroblasts of patients with congenital dyserythropoietic anemia. Blood 37:299-310

16. Crookston JH, Crookston MC, Burnie KL et al (1969) Hereditary erythroblastic multinuclearity associated with a positive acidifiedserum test: a type of congenital dyserythropoietic anaemia. Br J Haematol 17:11-26

17. Heimpel H, Anselstetter V, Chrobak L et al (2003) Congenital dyserythropoietic anemia type II: epidemiology, clinical appearance, and prognosis based on long-term observation. Blood 102: 4576-4581

18. Fukuda MN (1999) HEMPAS. Hereditary erythroblastic multinuclearity with positive acidified serum lysis test. Biochim Biophys Acta 1455:231-239

19. Wolff JA, Von Hofe FH (1951) Familial erythroid multinuclearity. Blood 6:1274-1283

20. Bergstrom I, Jacobsson L (1962) Hereditary benign erythroreticulosis. Blood 19:296-303

21. Iolascon A, Heimpel H, Wahlin A, Tamary H (2013) Congenital dyserythropoietic anemias: molecular insights and diagnostic approach. Blood 122:2162-2166

22. Tamary H, Dgany O, Proust A et al (2005) Clinical and molecular variability in congenital dyserythropoietic anaemia type I. Br J Haematol 130:628-634

23. Iolascon A, Esposito MR, Russo R (2012) Clinical aspects and pathogenesis of congenital dyserythropoietic anemias: from morphology to molecular approach. Haematologica 97:1786-1794. doi:10.3324/haematol.2012.072207, Epub 2012 Oct 12

24. Schwarz K, Iolascon A, Verissimo F et al (2009) Mutations affecting the secretory COPII coat component SEC23B cause congenital dyserythropoietic anemia type II. Nat Genet 41:936-940

25. Arnaud L, Saison C, Helias V et al (2010) A dominant mutation in the gene encoding the erythroid transcription factor KLF1 causes a congenital dyserythropoietic anemia. Am J Hum Genet 87:721-727

26. Waye JS, Eng B (2015) Krüppel-like factor 1: hematologic phenotypes associated with KLF1 gene mutations. Int J Lab Hematol 37: 78-84

27. Burwick N, Shimamura A, Liu JM (2011) Non-Diamond Blackfan anemia disorders of ribosome function: Shwachman Diamond syndrome and 5q- syndrome. Semin Hematol 48:136-143. doi:10. 1053/j.seminhematol.2011.01.002

28. Smith OP, Hann IM, Chessells JM, Reeves BR, Milla P (1996) Haematological abnormalities in Shwachman-Diamond syndrome. Br J Haematol 94:279-284

29. Shimamura A, Alter BP (2010) Pathophysiology and management of inherited bone marrow failure syndromes. Blood Rev 24: $101-122$

30. Soulier J (2011) Fanconi anemia. ASH Education Program Book 2011:492-497

31. Alter BP, Giri N, Savage SA, Rosenberg PS (2015) Telomere length in inherited bone marrow failure syndromes. Haematologica 100(1):49-54 